ISSN: 2386-3919 - e-ISSN: 2386-3927

DOI: https://doi.org/10.14201/et20173518198

\title{
DERECHOS HUMANOS Y EDUCACIÓN: EVALUACIÓN CONTINUA A TRAVÉS DE LA RED
}

\section{Human rights and education: continuous assessment through the Internet}

\author{
Isabel OrTega SÁncheZ*, María José AlBert GómeZ*** y María García PÉREZ**** \\ *UNED. Correo-e: iortega@edu.uned.es \\ * UNED. Correo-e: mjalbert@edu.uned.es \\ *** UNED. Correo-e: mgarcia@edu.uned.es
}

Recibido: 25/01/2016; Aceptado: 15/03/2017; Publicado: 31/05/2017

Ref. Bibl. ISABEL ORTEGA SÁNCHEZ, MARÍA JOSÉ ALBERT GÓMEZ y MARÍA GARCÍA PÉREZ. Derechos humanos y educación: evaluación continua a través de la Red. Enseñanza \& Teaching, 35, 1-2017, 81-98.

RESUMEN: La evaluación continua de calidad es uno de los retos actuales en la enseñanza universitaria para su adaptación al Espacio Europeo de Educación Superior. Dentro de la educación a distancia, la adquisición de competencias a través de la evaluación continua se debe diseñar teniendo en cuenta el contexto educativo y la utilización de las tecnologías. Nuestra investigación se centra en la asignatura de "Derechos Humanos y Educación" del Grado en Educación Social y del Grado en Pedagogía en la UNED. Los futuros pedagogos y educadores sociales tienen que adquirir los conocimientos básicos sobre Derechos Humanos y Educación y las competencias necesarias para transmitir y llevar a la práctica lo aprendido. Diseñamos una Red de Innovación Docente para investigar sobre la adquisición de competencias a través de una evaluación continua a distancia. Con esta investigación conocemos cómo valoran los estudiantes la adquisición de competencias a través de la realización de diferentes actividades de evaluación continua dentro del curso virtual de la asignatura. Para ello, utilizamos un cuestionario anónimo para los estudiantes con una muestra de 105 estudiantes. Los estudiantes valoran positivamente las actividades de evaluación continua, pero es necesario el replanteamiento del diseño de las actividades para fomentar la motivación, la adquisición de competencias 
transversales, el aprendizaje colaborativo y el valor que debe tener en sus aprendizajes. Posteriormente, describimos un modelo de evaluación continua de calidad a través de las tecnologías.

Palabras clave: evaluación continua; derechos humanos y educación; competencias; curso virtual.

SUMMARY: The quality continuous assessment is one of the current challenges in the university education for its adaptation to the European Higher Education Area. Inside the education over a distance the skills acquisition across the continuous assessment, it is necessary to design bearing in mind the educational context and the use of the technologies. Our investigation centers on the subject of «Human rights and Education" of Degree in Social Education and of the Degree in Pedagogy in the UNED. The future pedagogues and social educators have to acquire the basic knowledge on Human rights and Education and the necessary skills to transmit and to take to the practice the learned. We design a Network of Teaching Innovation to investigate on the skills acquisition across a continuous assessment over a distance. With this investigation we know how the students value the skills acquisition across the achievement of different activities of continuous assessment inside the virtual course of the subject. For it, we use an anonymous questionnaire for the students with a sample of 105 students. The students value positively the continuous assessment activities but there is necessary the reconsideration of the design of the activities to encourage the motivation, the acquisition of transverse skills, learning collaborative and the value that it must have in its learnings. Later we describe a quality continuous assessment model across the technologies.

Key words: continuous assessment; human rights and education; skills; virtual course.

\section{INTRODUCCIÓN}

Los conocimientos sobre Derechos Humanos y Educación son relevantes para cualquier educador si queremos conseguir desarrollar una sociedad ética y cada vez más humana donde sea posible una convivencia respetuosa y pacífica.

Los derechos humanos y su relación con la educación se pueden aprender en diferentes contextos donde se incida no solo en la adquisición de conocimientos, sino también en la adquisición de habilidades y valores. Por lo tanto, es necesaria una educación de los derechos humanos basada en competencias.

La adquisición de las competencias esenciales en el campo de los Derechos Humanos y la Educación va mucho más allá de la simple adquisición de conocimientos sobre los Derechos Humanos, sino que es de vital importancia que estos futuros pedagogos y educadores sociales adquieran nuevas habilidades, valores y sean capaces de interiorizar y poner en práctica las competencias específicas como son aquellas requeridas dentro de la Declaración Universal de los Derechos Humanos (DUDH). 


\section{MARCO DE LA INVESTIGACIÓN}

\subsection{Evaluación continua a través de las tecnologías}

Las tecnologías nos ofrecen un abanico amplio de oportunidades de aprendizaje en tiempo real o diferido, llegando a multitud de personas y desarrollando nuevas posibilidades en la adquisición de conocimientos y en la evaluación continua. Tosina, Díaz y Blázquez (2012: 161) nos explican cómo «debemos utilizar estas tecnologías para innovar y no para reproducir modelos tradicionales poco efectivos".

Es necesaria la incorporación de cambios innovadores en los procesos de enseñanza-aprendizaje mejorando la evaluación continua a través de las tecnologías.

La Universidad Nacional de Educación a Distancia (UNED) utiliza las tecnologías como medios facilitadores del aprendizaje de sus estudiantes. Se ofrece la posibilidad de una enseñanza a distancia de calidad que combina materiales didácticos actualizados y una plataforma virtual con posibilidades de comunicación en tiempo real y diferido y una evaluación continua que permite a los estudiantes el desarrollo del aprendizaje autónomo, ubicuo y permanente.

Durante estos años, se ha diseñado la asignatura de Derechos Humanos y Educación para que los educadores sociales y pedagogos adquieran los conocimientos necesarios sobre los Derechos Humanos y la Educación y las competencias necesarias para transmitir y llevar a la práctica lo aprendido en los contextos educativos y sociales. También hemos diseñado un curso virtual desde el que se realizan las actividades de evaluación continua, los procesos de comunicación y el desarrollo de competencias como la alfabetización virtual.

La evaluación continua a distancia es un reto en la formación ya que conlleva la valoración formativa de los estudiantes a través de medios tecnológicos. Debemos diseñar las actividades de evaluación continua con las tecnologías adecuadas para poder generar una evaluación de calidad. Como nos señala Gil (2015: 110) «uno de los retos con el que nos enfrentamos es el de la adaptación de los contenidos, metodología, procedimientos y material a los nuevos requerimientos del aprendizaje en el Marco del Espacio Europeo de Educación Superior, aprovechando para ello los recursos y herramientas tecnológicas que se nos ofrecen las distintas plataformas educativas».

Las tecnologías nos ofrecen la posibilidad de aprender a distancia, de forma flexible y ubicua, desarrollar nuevos espacios de formación y modelos innovadores de evaluación que permitan el desarrollo de competencias relacionadas con los resultados de aprendizaje que pretendemos conseguir, así como éxitos en el aprendizaje.

La evaluación electrónica (e-assessment) se tiene que planificar seleccionando de forma adecuada las tecnologías que generen: motivación en los estudiantes, aprendizaje autónomo, colaborativo, desarrollo de competencias y mejora del rendimiento. Tenemos que tener muy en cuenta la formación del profesorado como guía en el aprendizaje a través de una evaluación continua donde se necesita 
un feedback constante y adecuado y la motivación como ejes de su intervención orientadora y motivadora.

\subsection{Valoración de la evaluación continua: incidencia en el aprendizaje}

Para la adaptación al EEEs es necesario un cambio en la concepción que tienen los estudiantes sobre la evaluación a través del desarrollo de una evaluación continua que mejore los resultados de aprendizaje. Ricoy y Fernández-Rodríguez (2013: 337) nos explican cómo «queda patente la analogía que establecen los estudiantes universitarios entre la evaluación y la realización de exámenes». El cambio en la concepción de evaluación hacia un conocimiento sobre lo que es la evaluación continua dependerá de la formación del profesorado y la puesta en práctica de una evaluación continua real que aporte beneficios en el aprendizaje de los estudiantes.

A través de numerosos estudios se ha corroborado que los estudiantes perciben positivamente la evaluación continua. Segalés, Brumos, Torres, Aran, Rull, Falgueras y Rubinat (2015: 11) nos explican cómo «los estudiantes perciben muy favorablemente las actividades de evaluación continua». Otros autores describen cómo con la evaluación continua a través de las tecnologías se agilizan los procesos. Ji, García Seoane, Vázquez, Flores y Álvarez (2014: 5) detallan que «se logra una gestión más rápida, automatizada y transparente a la hora de procesar las evaluaciones y calcular las calificaciones».

La forma de evaluar condiciona los aprendizajes de los estudiantes promoviendo un aprendizaje autónomo o colaborativo, impulsando o disminuyendo la motivación, mejorando o perjudicando el rendimiento de los estudiantes. Arribas (2012: 3) nos explica cómo «la forma de evaluación condiciona el modo de aprendizaje del alumno e incide directamente sobre los aspectos emotivos del mismo: la motivación, el estilo atribucional, la autoeficacia, la autoestima, etc.».

La evaluación formativa incide en el aprendizaje y en el rendimiento académico, entendido este último como nos explican Aranda, Pastor, Oliva y Romero (2013: 24): «el rendimiento académico es la valoración de la capacidad de respuesta del individuo con la que se estima lo aprendido tras un proceso formativon. Arribas (2012: 11) nos resalta cómo «el sistema de evaluación y calificación empleado tiene una enorme incidencia sobre el rendimiento académico siendo la forma de evaluación continua la que propicia los mejores resultados en el rendimiento y en las calificaciones obtenidas». Aranda, Pastor, Oliva y Romero (2013: 32) nos detallan cómo «con la utilización de sistemas de evaluación formativa influyen en la mejora del rendimiento académico y es más alto con la evaluación continua o mixta que con el examen final como reflejo de un mejor proceso de aprendizaje». Parrilla, Rullán y Bauzà (2013: 41) nos explican cómo «los estudiantes que han optado por el itinerario de evaluación continua han obtenido una mejor calificación y, por tanto, un mejor resultado de aprendizaje en términos de los conocimientos adquiridos".

Por lo tanto, la evaluación continua mejora el rendimiento de los estudiantes y los resultados de aprendizaje, pero existen muchos tipos de evaluación continua 
que incidirán de forma diferente en los estudiantes y dependerá de muchos factores a tener en cuenta antes de planificar una evaluación de continua de calidad a través de las tecnologías.

\subsection{Evaluación a través del trabajo autónomo y colaborativo}

El desarrollo del aprendizaje autónomo en la educación a distancia se realiza con la adquisición de una serie de competencias a través del trabajo individual, estudio, actividades de autoevaluación y el desarrollo de competencias comunicativas y tecnológicas que facilitan el aprendizaje a distancia a través de Internet. Salvador, Villach, Saíz y Llanos (2007: 785) nos explican cómo «el proceso de convergencia europea ha impulsado también la implementación de metodologías docentes centradas el trabajo autónomo de los estudiantes. Para ello, se considera necesario que los estudiantes dispongan de competencias que les permitan regular el aprendizaje individual y de grupo".

La evaluación continua puede beneficiar el desarrollo del aprendizaje autónomo si en su planificación tenemos en cuenta este objetivo.

El desarrollo del aprendizaje colaborativo a través de las actividades de evaluación continua es más complejo debido a la falta de adquisición de competencias para este tipo de aprendizaje y de algunas dificultades que se tienen al regular estos aprendizajes en los estudiantes. En la mayoría de los casos, solo los foros de debates dentro de un curso virtual fomentan el intercambio de conocimientos e ideas, la reflexión y la construcción conjunta del aprendizaje.

Casi siempre las actividades de evaluación a distancia son individuales y las grupales son difíciles de plantear en una educación a distancia a través de Internet debido a los problemas de comunicación en los grupos de estudiantes y a la gestión de estos grupos por parte del profesorado para conseguir éxitos en sus aprendizajes.

Resaltamos también la necesidad de organizar de forma adecuada la ratio profesor/estudiantes cuando se proponen actividades grupales dentro de una evaluación continua a través de las tecnologías. El número de estudiantes dentro del grupo no debe ser muy elevado para poder llegar a acuerdos y establecer procesos de comunicación dinámicos con retroalimentaciones asiduas por parte del profesor.

La evaluación continua a través del trabajo colaborativo no se desarrolla con asiduidad debido a la complejidad de la evaluación. En los diseños pedagógicos de los cursos virtuales se observa un diseño poco colaborativo debido a las dificultades que este plantea al tener que coordinar, a veces, grandes cantidades de estudiantes a través de la Red. Son más frecuentes test de autoevaluación, foros de debates y actividades generales online.

Como señala Zañartu (2002), las actividades colaborativas mantienen características importantes sobre todo cuando se habla del aprendizaje apoyado por las tecnologías y que son: la interactividad, grado de sincronización (sincrónica y asincrónica) y la negociación. 
Aunque somos conscientes de las dificultades del trabajo colaborativo debemos tener en cuenta su importancia dentro del aprendizaje al ser muy valorado por los estudiantes e influir de forma positiva en el rendimiento. En el estudio planteado por Rebollo Catalán, García Pérez, Buzón García y Barragán Sánchez (2013: 122) «los resultados muestran una valoración positiva de los estudiantes acerca de la metodología de aprendizaje colaborativo experimentada en su conjunto».

\section{OBjetivos}

\subsection{Objetivo general}

Diseñamos una Red de Innovación Docente para investigar sobre la adquisición de competencias a través de una evaluación continua a distancia. Nos propusimos la adquisición de competencias a través de la realización de diferentes actividades y con esta red queremos conocer cómo el estudiante valora su realización para poder mejorarlas, modificarlas o crear otras nuevas atendiendo a la adquisición de competencias.

Desde esta perspectiva, el objetivo general de esta red se centró en el fomento y desarrollo del aprendizaje activo, colaborativo y significativo como elemento motivador para el desarrollo de las competencias que son necesarias para el alumno en su futuro profesional a través de la evaluación continua.

\subsection{Objetivos específicos}

Con lo cual, a través de esta red proponemos los siguientes objetivos específicos:

1. Mejorar los procesos de evaluación continua valorando la adquisición de conocimientos y de competencias por parte de los estudiantes.

2. Favorecer el aprendizaje continuo real consiguiendo la autonomía en el aprendizaje.

3. Motivar al estudiante a través de la evaluación continua utilizando diferentes herramientas.

4. DisEÑO Y MÉTODOS DE LA INVESTIGACIÓN

\subsection{Muestra}

El número de estudiantes que participaron fue de 105 alumnos y alumnas del primer año del Grado de Educación y del Grado de Pedagogía. 


\subsection{Diseño y planificación del trabajo}

Con los fundamentos teóricos aportados por la bibliografía básica se estructuran las actividades que pretenden enriquecer y mejorar la calidad educativa de la asignatura, pero también crear un entorno de aprendizaje donde el alumno reflexione, se produzca la interiorización de los contenidos y el desarrollo de competencias.

Los recursos virtuales utilizados facilitan los procesos de comunicación y la resolución de dudas, fomentan un aprendizaje significativo y la motivación atendiendo a las necesidades e intereses de los estudiantes, posibilitando el desarrollo del aprendizaje autónomo, colaborativo y la adquisición de competencias.

Se planificaron la actividades propuestas, tanto grupales como individuales, que promueven el desarrollo y adquisición de competencias de carácter genérico. Estas actividades también facilitan el trabajo autónomo y colaborativo de los estudiantes, reforzando el desarrollo de competencias de diversa índole, pero además teniendo en cuenta las competencias necesarias para esta asignatura en concreto, Derechos Humanos y Educación.

Tras la identificación de cada una de las actividades se construyó un cuestionario anónimo en línea realizado a través de google doc y colgado en el foro de consultas generales de la plataforma de la asignatura Derechos Humanos y Educación.

Las preguntas eran de elección múltiple aunque también se crearon preguntas abiertas donde los participantes de la investigación han tenido la oportunidad de ofrecer sus impresiones, expectativas, demandas y soluciones sobre aspectos concretos de la evaluación continua.

Se recogieron las valoraciones de una muestra de 105 estudiantes sobre las actividades de evaluación continua y la evaluación final y a través de un análisis descriptivo cuantitativo y cualitativo de los resultados se obtuvieron interesantes datos para valorar el nivel de satisfacción de los estudiantes sobre las actividades de evaluación continua.

\section{ANÁlisis DE LOS RESULTADOS}

Nuestra investigación es de carácter descriptivo-interpretativo mediante el empleo de un cuestionario que permite el análisis de la evaluación continua.

\subsection{Análisis cuantitativo}

\subsubsection{Tipo de evaluación}

En el siguiente gráfico podemos ver el porcentaje de las respuestas recogidas sobre los tipos de evaluación desarrolladas en la asignatura. 


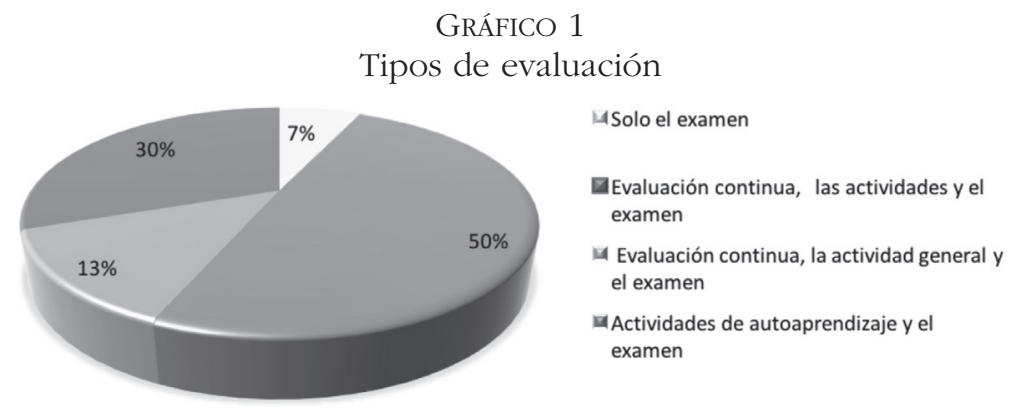

El 50\% de los estudiantes desarrollan la modalidad de evaluación continua a través de la realización de la actividad general que puntuaba en la nota final, las actividades de autoaprendizaje que no eran obligatorias y se centraban especialmente en desarrollar las competencias específicas que se buscaban alcanzar en esta asignatura llamada "Derechos Humanos y Educación" y el examen. El 30\% de los estudiantes ha elegido el modelo de evaluación continua solo con las actividades de autoaprendizaje y el examen. Solo el 13\% ha elegido la modalidad de evaluación continua con la realización de la actividad general que sí puntuaba en la nota final y el examen.

Los resultados nos muestran que la mayoría de los estudiantes (93\%) han elegido la evaluación continua y han realizado todas las actividades, tanto la actividad general, como las actividades de autoaprendizaje. Pero, además, hay que tener en cuenta que las actividades de autoaprendizaje no puntuaban para la calificación final, mientas que la actividad general sí. Un número muy reducido de estudiantes (7\%) solo se van a presentar al examen sin realizar la evaluación continua.

Podemos resaltar que el alumno prefiere la evaluación continua, es consciente de su importancia y cómo incide positivamente en su aprendizaje.

\subsubsection{Actividad general}

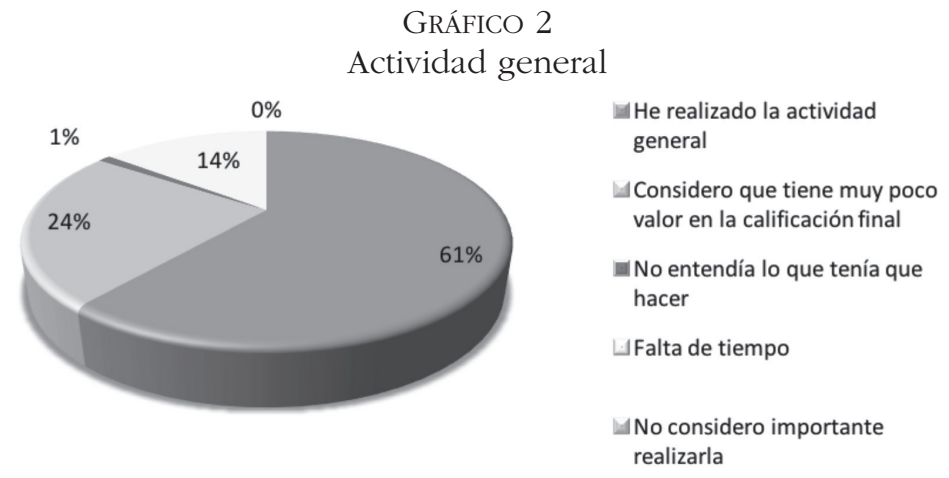


Lo primero que se puede observar de estos datos es que los resultados obtenidos sobre la actividad general van en consonancia con los resultados obtenidos en el tipo de evaluación realizada, lo que nos ofrece una validez de contenido.

La gran mayoría de estudiantes (61\%) ha realizado la actividad general. Un dato relevante es que ninguno de ellos considera que esta actividad no es importante, sino que sus razones para no realizarla han sido por falta de tiempo (14\%) o porque han considerado que tenía muy poco valor en la calificación final (24\%). Tan solo un $1 \%$ de los estudiantes no habían entendido lo que tenían que hacer, y este porcentaje tan bajo tratándose de estudiantes de primero de grado y del primer semestre es interpretado por el equipo docente como un gran logro metodológico.

5.1.3. Realización de actividades de autoaprendizaje

GRÁFICO 3

Actividades de autoaprendizaje

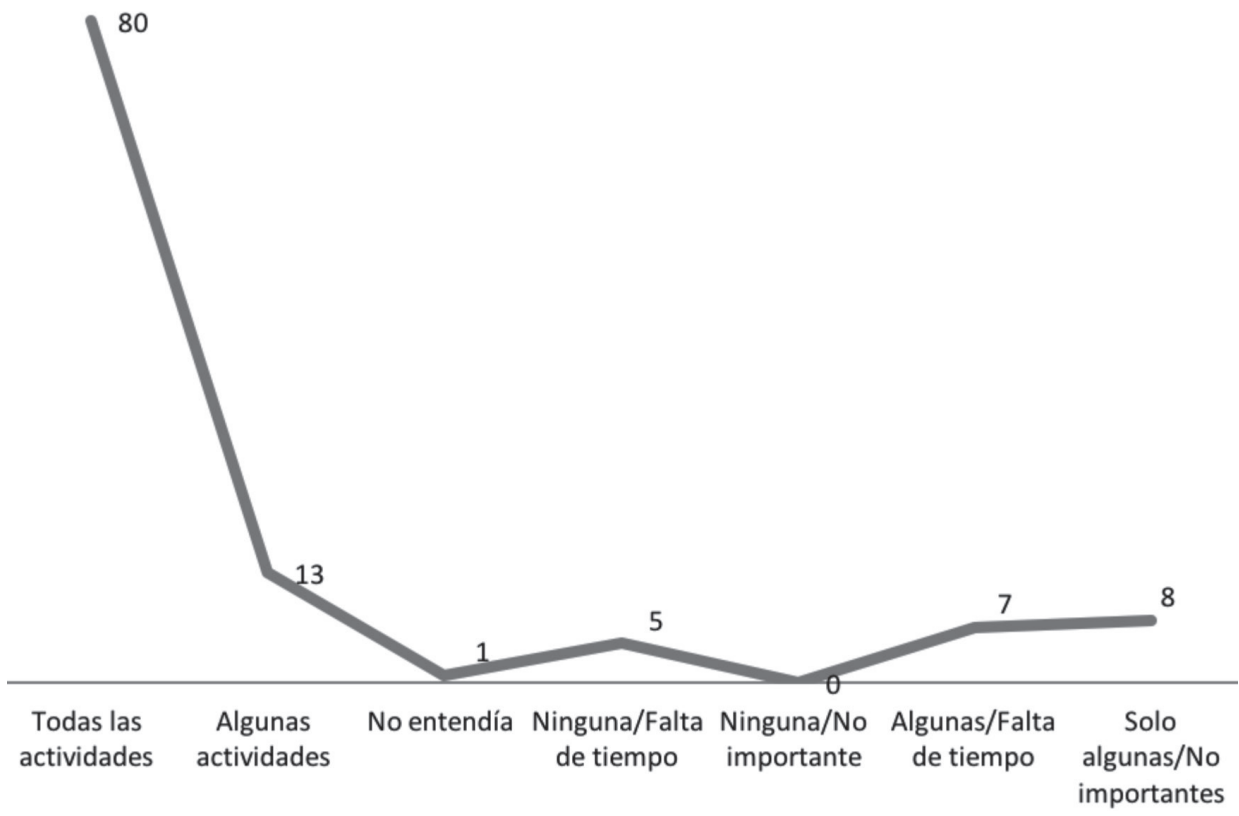

Las actividades de autoaprendizaje se centran en desarrollar las competencias específicas de la asignatura Derechos Humanos y Educación, pero no tienen valor en la calificación final. Los estudiantes tienen esta información, por lo que es importante destacar que el $80 \%$ de los estudiantes han realizado las actividades de autoaprendizaje ya que mejoran su aprendizaje y el desarrollo de competencias. Resaltamos cómo el 7\% de los estudiantes no las ha realizado todas por falta de tiempo y otros 
consideraban que algunas no eran importantes (8\%). Solo un 5\% de los estudiantes no ha realizado las actividades por falta de tiempo y un 1\% porque no las entendía.

Los resultados nos aportan una visión diferente de las actividades de algunos temas que han sido realizadas por menos estudiantes y tendremos que valorar el diseño de algunas actividades y la generación de nuevos procesos de motivación en el curso siguiente.

\subsubsection{Valoración del sistema de actividades}

Para conocer las valoraciones de los estudiantes sobre las actividades se ha utilizado una escala Likert. Se han preguntado cuestiones muy específicas sobre las actividades y el interés que tiene el alumno. Se evalúa la motivación que las actividades de autoaprendizaje y la actividad general provocan en los estudiantes y la opinión de los estudiantes respecto a su utilidad.

TABLA 1

Valoraciones de las actividades de autoaprendizaje y la actividad general

\begin{tabular}{|c|c|c|c|c|c|}
\hline AFIRMACIONES & $\begin{array}{c}\text { MUY DE } \\
\text { ACUERDO }\end{array}$ & $\begin{array}{c}\text { DE } \\
\text { ACUERDO }\end{array}$ & INDIFERENTE & $\begin{array}{c}\text { EN } \\
\text { DESACUERDO }\end{array}$ & $\begin{array}{c}\text { MUY EN } \\
\text { DESACUERDO }\end{array}$ \\
\hline $\begin{array}{c}\text { Todas las actividades } \\
\text { son imprescindibles para } \\
\text { mejorar el aprendizaje }\end{array}$ & $78 \%$ & $10 \%$ & $7 \%$ & $4 \%$ & $1 \%$ \\
\hline $\begin{array}{c}\text { Las actividades de } \\
\text { autoaprendizaje } \\
\text { no son útiles }\end{array}$ & $0 \%$ & $1 \%$ & $5 \%$ & $71 \%$ & $23 \%$ \\
\hline $\begin{array}{c}\text { La actividad general } \\
\text { no es útil }\end{array}$ & $4 \%$ & $2 \%$ & $4 \%$ & $38 \%$ & $52 \%$ \\
\hline $\begin{array}{c}\text { Las actividades de } \\
\text { autoaprendizaje son } \\
\text { imprescindibles para } \\
\text { mejorar el aprendizaje }\end{array}$ & $82 \%$ & $5 \%$ & $8 \%$ & $2 \%$ & $3 \%$ \\
\hline $\begin{array}{c}\text { La actividad general } \\
\text { es imprescindible para } \\
\text { mejorar el aprendizaje }\end{array}$ & $65 \%$ & $23 \%$ & $12 \%$ & $5 \%$ & $0 \%$ \\
\hline $\begin{array}{c}\text { Se puede identificar } \\
\text { con cada actividad } \\
\text { el tema que se } \\
\text { pretende desarrollar }\end{array}$ & $52 \%$ & $27 \%$ & $11 \%$ & $7 \%$ & $3 \%$ \\
\hline $\begin{array}{c}\text { Todas las actividades } \\
\text { me han motivado para } \\
\text { seguir aprendiendo } \\
\text { en esta asignatura }\end{array}$ & $13 \%$ & $35 \%$ & $23 \%$ & $14 \%$ & $15 \%$ \\
\hline
\end{tabular}


Teniendo en cuenta las dos variables que resaltábamos, motivación y utilidad, analizamos los resultados obtenidos. Según estos datos para los estudiantes las actividades son importantes para mejorar el aprendizaje (88\%). Las actividades de autoaprendizaje son útiles (94\%) y mejoran el aprendizaje (87\%). La actividad general tienen una gran utilidad (90\%) y mejora el aprendizaje (88\%). Una gran mayoría de estudiantes están de acuerdo en que se identifica cada actividad con el tema que se quiere desarrollar (79\%). También resaltamos que todas las actividades han motivado para seguir aprendiendo (48\%) y no han motivado a algunos estudiantes (29\%). Este dato está relacionado con la no realización de algunas actividades por falta de motivación.

Resaltamos la gran utilidad de las actividades y cómo mejora el aprendizaje. No todas las actividades han sido motivadoras por lo que es necesario un diseño innovador de las mismas.

5.1.5. Valoración del sistema de evaluación continua

TABLA 2

Valoraciones sistema de evaluación continua

\begin{tabular}{|c|c|c|c|c|c|}
\hline $\begin{array}{c}\text { AFIRMACIONES. } \\
\text { CONSIDERO NECESARIO QUE: }\end{array}$ & $\begin{array}{c}\text { MUY DE } \\
\text { ACUERDO }\end{array}$ & $\begin{array}{c}\text { DE } \\
\text { ACUERDO }\end{array}$ & INDIFERENTE & $\begin{array}{c}\text { EN } \\
\text { DESACUERDO }\end{array}$ & $\begin{array}{c}\text { MUY EN } \\
\text { DESACUERDO }\end{array}$ \\
\hline $\begin{array}{c}\text { Las actividades siempre } \\
\text { puntúen aunque no se } \\
\text { tenga un } 5 \text { en el examen }\end{array}$ & $89 \%$ & $6 \%$ & $5 \%$ & $0 \%$ & $0 \%$ \\
\hline $\begin{array}{c}\text { Todas las actividades } \\
\text { sean obligatorias }\end{array}$ & $0 \%$ & $16 \%$ & $5 \%$ & $45 \%$ & $34 \%$ \\
\hline $\begin{array}{c}\text { Solo puntúe en la } \\
\text { calificación final la } \\
\text { actividad general }\end{array}$ & $0 \%$ & $7 \%$ & $5 \%$ & $23 \%$ & $65 \%$ \\
\hline $\begin{array}{c}\text { Puntúe la actividad } \\
\text { general en mayor medida } \\
\text { en la calificación final } \\
\text { pero que sea obligatoria }\end{array}$ & $3 \%$ & $0 \%$ & $4 \%$ & $64 \%$ & $29 \%$ \\
\hline $\begin{array}{c}\text { Todas las actividades } \\
\text { sean voluntarias, pero } \\
\text { que puntúen en la } \\
\text { calificación final a partir } \\
\text { de un 5 en el examen }\end{array}$ & $9 \%$ & $36 \%$ & $2 \%$ & $51 \%$ & $2 \%$ \\
\hline $\begin{array}{c}\text { El peso de la actividades } \\
\text { en la calificación es justo } \\
\text { respecto al peso que se le } \\
\text { da en la calificación final }\end{array}$ & $11 \%$ & $14 \%$ & $2 \%$ & $46 \%$ & $27 \%$ \\
\hline
\end{tabular}


Los estudiantes buscan que todas las actividades puntúen aunque no se tenga aprobado el examen (95\%). Un 88\% desean que puntúe solo la actividad general en la calificación final y un 93\% quieren que la actividad general puntúe más y siga siendo obligatoria. También demandan que las actividades tengan un mayor peso en la calificación final (73\%),

Otra de las cuestiones importantes que surgen es que los estudiantes no desean la obligatoriedad de las actividades (79\%) y entienden que deban ser voluntarias para que se adapten a todas las problemáticas que puedan tener los diferentes estudiantes de la UNED.

\subsection{Análisis cualitativo. Valoraciones generales sobre el sistema de evaluación}

Cuando hemos analizado el contenido de las respuestas en las preguntas abiertas, todas tratan sobre la misma temática, la importancia del peso de las actividades en la evaluación final.

El 30\% de las respuestas indican que los estudiantes no quieren que las actividades sean obligatorias:

- Considero que todas las actividades de autoaprendizaje y la actividad general son muy interesantes y a mí particularmente me han ayudado mucho en esta asignatura, pero no deben ser obligatorias por que no todo el mundo dispone del mismo tiempo para poder hacerlas.

- Con respecto a las actividades, pienso que está muy bien que sean voluntarias, ya que en mi caso, aunque me hubiera gustado hacer la actividad general y no he podido por falta de tiempo (Trabajo, casa, hijos pequeños...), no podría presentarme a examen.

- El sistema me parece adecuado, las actividades voluntarias suponen parte del aprendizaje, sin embargo conllevan de mucho tiempo del que todas las personas no disponen. Estoy de acuerdo con el sistema actual.

- Con respecto a las actividades, pienso que está muy bien que algunas sean voluntarias, ya que en mi caso, aunque me hubiera gustado hacer la actividad general y no he podido por falta de tiempo y no podría presentarme a examen.

Y el 70\% de las respuestas se refieren a la importancia que tiene la realización de la actividad general en la nota final:

- Para que la evaluación sea realmente continua, las actividades generales deberían tener más peso en la nota final.

- Creo que la valoración de la actividad general debería ser un poco más alta, ya que conlleva mucho tiempo y esfuerzo, aunque también reconozco que se aprende mucho más haciéndola. La persona que realiza el trabajo llegará a ser más consciente de lo que transmite la asignatura y probablemente podrá transferir mejor sus conocimientos no solo de forma teórica sino también con su actitud.

En conclusión, la parte cualitativa de esta investigación nos muestra la importancia que tiene para los estudiantes la evaluación continua, pero también ellos 
quieren un reconocimiento mayor de su trabajo en la calificación final y la no obligatoriedad de las actividades propuestas.

\section{DisCUSIÓN Y CONCLUSIONES}

Resaltamos cómo la planificación de las diferentes actividades que tienen como fin la formación integral de los estudiantes mediante la potenciación y adquisición de diferentes competencias supone un nuevo reto para la práctica pedagógica de los docentes, puesto que tenemos que reformular el modelo de enseñanza que hasta el momento venían aplicando antes del Espacio Europeo de Educación Superior (EEES). En este sentido, los diferentes tipos de actividades que se analizaron han experimentado una evolución progresiva hacia metodologías más participativas, centradas en el discente y apoyadas en el uso de herramientas tecnológicas.

Como afirman Del Moral y Villalustre (2009), al igual que señalan nuestros resultados en esta investigación, mediante la incorporación de actividades colaborativas e individuales se han potenciado y desarrollado toda una serie de competencias, atendiendo a los nuevos requerimientos impulsados por el proceso de Convergencia Europea.

A través de los resultados obtenidos en esta investigación se deduce que los estudiantes consideran que las actividades de evaluación continua son importantes para el desarrollo de sus competencias y para ampliar sus conocimientos en la materia de los Derechos Humanos y Educación.

Y aunque los resultados han sido excelentes según nuestra investigación, los estudiantes deben desarrollar nuevas competencias que supongan una actitud más activa y comprometida con su propio aprendizaje, deben tener la capacidad de una mayor adaptación a los cambios y ser verdaderos autónomos de su aprendizaje. Por esta razón, las actividades deben centrarse en la figura del alumno, facilitándole el trabajo autónomo y el colaborativo, así como la adquisición tanto de competencias específicas propias de su perfil profesional, como las competencias transversales o genéricas (Del Moral y Villalustre, 2008).

Parrilla, Rullán y Bauzà (2013: 42) expresan cómo «los mejores resultados de la evaluación continua se deben a que motiva a los estudiantes a realizar dicho esfuerzo». La motivación es uno de los ejes para desarrollar una evaluación continua de calidad y con los resultados de nuestra investigación afirmamos que es necesario un cambio en el diseño de algunas actividades para elevar la motivación de nuestros estudiantes.

Corroboramos cómo los estudiantes realizan las actividades de autoaprendizaje y la actividad general porque las consideran útiles y mejoran su aprendizaje. Cebrián y García (2015: 252) expresan cómo la evaluación continua «ayuda a mejorar el resultado académico de los estudiantes».

A continuación describimos los factores relevantes a tener en cuenta para diseñar una evaluación continua de calidad a través de las tecnologías teniendo en cuenta la planificación de la evaluación, la utilización de los recursos tecnológicos adecuados, la motivación a través del seguimiento del profesor y la 
retroalimentación, una metodología innovadora y contextualizada, el diseño de actividades online y procesos de comunicación interactivos.

TABLA 3

Factores relevantes en la evaluación continua a través de las tecnologías

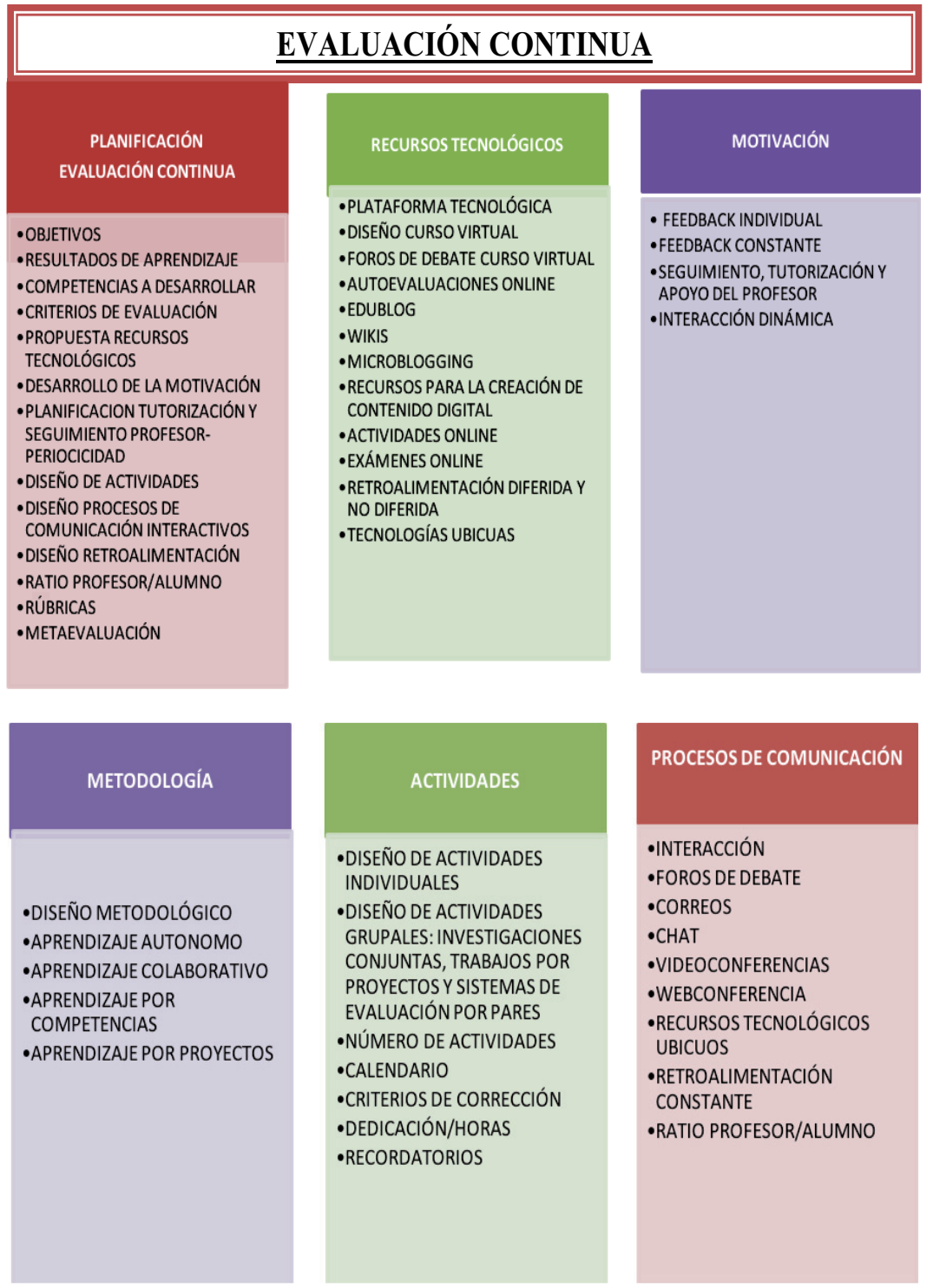

FUENTE: Elaboración propia. 
En la evaluación continua los factores expuestos son indispensables junto con el uso adecuado de las tecnologías para facilitar la evaluación continua online de un gran número de estudiantes. Las seis grandes columnas que deben sustentar una evaluación continua de calidad son la planificación detallada de la evaluación continua, desarrollando los objetivos vinculados a los resultados de aprendizaje y a las competencias que deseamos desarrollar; la utilización de los recursos tecnológicos necesarios según el contexto educativo; el desarrollo de la motivación a través de los procesos de tutorización, interacción, seguimiento y apoyo constante del profesor; el diseño de una metodología acorde con los objetivos y los resultados de aprendizaje propuestos; la creación de actividades a través de las tecnologías que fomenten el aprendizaje autónomo, colaborativo y el tiempo de dedicación; la metodología específica según el proceso de evaluación que queremos desarrollar; y, por último, debemos tener en cuenta la importancia de la comunicación a través de las tecnologías para mejorar los procesos de enseñanza-aprendizaje, la motivación y la evaluación continua.

Como nos explican Olmos Migueláñez y Rodríguez Conde (2012: 135) «una de las herramientas más útiles a disposición del docente para influir positivamente en el proceso de aprendizaje es la capacidad de elegir el diseño de estrategia de evaluación más adecuado a los resultados de aprendizaje que intenta promover en sus estudiantes, sin olvidar las aportaciones de las tecnologías en este campo".

El diseño metodológico de la evaluación continua será la base para el diseño dentro del curso virtual. Después del diseño nos centraremos en la elección de las actividades, los procesos de comunicación e interacción con la utilización de las herramientas tecnológicas necesarias, el desarrollo de la motivación a través de los procesos de tutorización mediante el seguimiento y la retroalimentación para la mejora de los resultados de aprendizaje a través de la evaluación continua.

Resaltamos la importancia de utilizar dentro de la evaluación continua el aprendizaje basado en competencias ya que es necesario que los estudiantes desarrollen los conocimientos, habilidades y actitudes que necesitarán después en su vida laboral. Zea, Medina-Medina, Vela, Rodríguez, López-Arcos, Delgado y Polo (2015) nos explican cómo «el aprendizaje basado en competencias está orientado a la formación de los estudiantes con conocimientos que puedan aplicar de forma práctica para resolver problemas con calidad de tal forma que sean capaces de comprender, contextualizar y analizar las acciones que realizan".

Una evaluación continua de calidad que beneficie el rendimiento de los estudiantes está realizada por profesionales que hacen un seguimiento continuo del progreso y el aprendizaje de los estudiantes mediante un nivel alto de interactividad, con orientaciones y retroalimentaciones constantes durante los procesos de enseñanza-aprendizaje. Salvador, Villach, Saíz y Llanos (2007: 786) detallan que «el seguimiento, la tutorización y el apoyo del profesor al trabajo de los estudiantes es de una enorme importancia y constituye sin duda uno de los elementos fundamentales para el éxito de los procesos de enseñanza y aprendizaje».

Arrufat, Sánchez y Santiuste (2015: 91) explican: 
una serie de propuestas de mejora de los sistemas evaluativos en este formato de cursos masivos:

- Aumentar la variedad de herramientas de evaluación para una mayor adaptación a diferentes formas de aprendizaje.

- Acomodar el tipo de herramientas a los objetivos de aprendizaje.

- Orientar el sistema evaluativo hacia competencias y aprendizaje colaborativo.

- Implementar sistemas evaluativos que potencien la interactividad y el feedback entre los participantes.

- Potenciar el autoaprendizaje a través de la corrección automática de las pruebas y cuestionarios.

Consideramos también relevante el aprendizaje colaborativo formado por 5 estudiantes que realizan una parte del trabajo especificada y es evaluada por el profesor-tutor o a través de la evaluación por pares (otros compañeros son los evaluadores). La evaluación continua a través de esta actividad colaborativa permite el desarrollo de una evaluación continua en grupos cuando son gran cantidad de estudiantes y fomenta competencias como la reflexión conjunta, resolución de problemas, autonomía, empatía, mejora los procesos de comunicación, potencia la creatividad, se adquieren habilidades sociales y cognitivas.

Sevillano García y Colmena (2013: 179) nos explican que «se hace necesario que tanto en el ámbito de la formación universitaria como en la formación permanente se potencie la valoración educativa de las Nuevas Tecnologías de la Información y de la Comunicación en la labor profesional docente». La utilización de las tecnologías en los procesos de evaluación continua mejora el aprendizaje y facilita los procesos al evaluar gran cantidad de estudiantes, pero el profesorado debe estar formado para realizar cambios en la planificación y en el desarrollo de la evaluación continua generando procesos evaluativos innovadores adaptados a los entornos virtuales.

En la asignatura de Derechos Humanos y Educación tenemos el compromiso de enseñar la importancia que tienen los Derechos Humanos y la Educación como guía básica para el desarrollo de una sociedad pacífica donde el respeto a estos derechos sea la base de los comportamientos y las relaciones humanas. Seguiremos trabajando para conseguir mejorar la adquisición de competencias de forma innovadora, la evaluación continua y la adaptación a los cambios del mercado laboral, pero hemos conseguido la adquisición de las siguientes competencias: el desarrollo de procesos cognitivos superiores, autonomía, aprendizaje colaborativo e individual, el uso de las herramientas tecnológicas, el desarrollo de actitudes éticas de acuerdo con los principios deontológicos y el compromiso social, motivar a educar en derechos humanos, el diagnóstico de situaciones complejas que fundamenten el desarrollo de las acciones socioeducativas, la comprensión de los referentes teóricos, la concienciación de la importancia de los derechos humanos y la educación para la defensa de los derechos humanos y la generación de sociedades más humanas y democráticas. 


\section{REFERENCIAS BIBLIOGRÁFICAS}

Aranda, A. F.; Pastor, V. M. L.; Oliva, F. J. C. y Romero, R. (2013). La evaluación formativa en docencia universitaria y el rendimiento académico del alumnado. Aula Abierta, 41 (2), 23-34.

Arribas, J. M. (2012). El rendimiento académico en función del sistema de evaluación empleado. Revista Electrónica de Investigación y Evaluación Educativa, 18 (1), 1-15.

Arrufat, M. J. G.; Sánchez, V. G. y Santiuste, E. G. (2015). Tendencias en la evaluación del aprendizaje en cursos en línea masivos y abiertos. Educación XX1, 18 (2).

Cebrián, F. J. y García, R. L. (2015). Actividades de evaluación continua-correlación con la calificación de la prueba final y efecto sobre la calificación final. Evidencia en Administración y Dirección de Empresas. Revista Complutense de Educación, 26 (2), 241-254.

Del Moral, M. E. y Villalustre, L. (2008). Sviluppo di progetti collaborativi in corsi universitari a partire da un gioco di simulazione e da Wiki. Tecnologie Didattiche, 45, 3, 31-37.

Del Moral, M. E. y Villalustre, L. (2009). Modalidades de aprendizaje telemático y resultados interuniversitarios extrapolables al nuevo EEES (Proyecto MATRIX). Barcelona: Editorial Octaedro.

García, M. L. S. y Colmena, R. F. (2013). Formación inicial del profesorado en TICS: Un análisis de Castilla la Mancha. Profesorado. Revista de Currículum y Formación de Profesorado, 17 (3), 151-183.

Gil, J. J. S. (2015). Innovación pedagógica en educación continua: hacia un curso de metodología de investigación clínica en el marco de Espacio Europeo de Educación Superior usando la plataforma educativa Blackboard. RIESED-Revista Internacional de Estudios sobre Sistemas Educativos, 2 (4), 109-118.

Ji, Z.; García Seoane, J. J.; Vázquez, T.; Flores, R. y Álvarez, M. P. (2014). V Encuentro Internacional de Intercambio de Experiencias Innovadoras en la Docencia. Madrid.

Olmos Migueláñez, S. y Rodríguez Conde, M. J. (2012). Perspectiva tecnológica de la evaluación educativa en la universidad. Teoría de la Educación, 23 (1), 22.

Parrilla, J. C.; Rullán, X. O. y Bauzà, M. S. (2013). Evaluando la evaluación continua. @ tic. revistad'innovació educativa, 10, 33-43.

Rebollo Catalán, M.; García Pérez, R.; Buzón García, O. y Barragán Sánchez, R. (2013). Las comunidades virtuales como potencial pedagógico para el aprendizaje colaborativo a través de las Tic. Enseñanza \& Teaching: Revista Interuniversitaria de Didáctica, 30 (2), 105-126. Recuperado de http://campus.usal.es/ revistas_trabajo/index.php/0212-5374/ article/view/9317/9610.

Ricoy, M. C. y Fernández-Rodríguez, J. (2013). La percepción que tienen los estudiantes universitarios sobre la evaluación: Un estudio de caso. Educación XX1, 16 (2), 321-342.

Salvador, C. C.; Villach, M. J. R.; Saíz, R. M. M. y Llanos, M. N. (2007). Evaluación continua y ayuda al aprendizaje. Análisis de una experiencia de innovación en educación superior con apoyo de las Tic. Electronic Journal of Research in Educational Psychology, 5 (13), 783-804.

Segalés, A. V.; Brumos, M. V. G.; Torres, R. M. N.; Aran, A. P.; Rull, J. O.; Falgueras, E. A. y Rubinat, M. T. (2015). Actividades de evaluación continua: percepción de los estudiantes. Un estudio multidisciplinar. Revista del Congrés Internacional de Docència Universitària i Innovació (CIDUI), (2).

Tosina, R. Y.; Díaz, L. A. y Blázquez, F. (2012). La e-evaluación de aprendizajes en educación superior a través de aulas virtuales síncronas. Comunicar: Revista Científica Iberoamericana de Comunicación y Educación, 39, 159-167. 
Zañartu, L. M. (2002). Aprendizaje colaborativo: una nueva forma de Diálogo Interpersonal $y$ en Red. [En línea]. Descargado el: 02/4/13. Disponible en http://contexto-educativo. com.ar/2003/4/nota-02.htm

Zea, N. P.; Medina-Medina, N.; Vela, F. L. G.; Rodríguez, P. P.; López-Arcos, J. R.; Delgado, M. P. N. y Polo, J. R. (2015). Evaluación continua para aprendizaje basado en competencias: Una propuesta para videojuegos educativos. IE Comunicaciones: Revista Iberoamericana de Informática Educativa, 21, 3. 